\title{
Prognostic significance of p16 in locoregionally advanced head and neck cancer treated with concurrent 5-fluorouracil, hydroxyurea, cetuximab and intensity-modulated radiation therapy
}

\author{
CHARLES C.L. TONG ${ }^{1,3 *}$, K.H. VINCENT LAU ${ }^{1 *}$, MICHAEL RIVERA $^{2}$, DAVID CANNAN $^{3}$, \\ JULIO AGUIRRE-GHISO $^{3}$, ANDREW G. SIKORA ${ }^{3}$, VISHAL GUPTA ${ }^{1}$, KEVIN FORSYTHE ${ }^{1}$, \\ ERIC C. KO ${ }^{1}$, KRZYSZTOF MISIUKIEWICZ ${ }^{4}$, VIVEK GURUDUTT ${ }^{3}$, MARITA S. TENG ${ }^{3}$, \\ STUART H. PACKER ${ }^{4}$, ERIC M. GENDEN ${ }^{3}$ and JOHNNY KAO ${ }^{5}$
}

\begin{abstract}
Departments of ${ }^{1}$ Radiation Oncology, ${ }^{2}$ Pathology, ${ }^{3}$ Otolaryngology-Head and Neck Surgery, and ${ }^{4}$ Medical Oncology, Mount Sinai School of Medicine, New York, NY; ${ }^{5}$ Florida Radiation Oncology Group, Brandon, FL, USA
\end{abstract}

Received November 30, 2011; Accepted January 4, 2012

DOI: $10.3892 /$ or.2012.1679

\begin{abstract}
A phase II trial was conducted to evaluate the tolerability and efficacy of incorporating cetuximab and simultaneous integrated boost intensity-modulated radiation therapy (SIB-IMRT) into a well-described 5-fluorouracil (5-FU) and hydroxyurea (HU)-based chemoradiation regimen. Patients with stage IVA-IVB or high-risk stage III squamous cell carcinomas were enrolled. Prior organ-conserving surgery or induction chemotherapy was allowed. IMRT was administered in $1.5 \mathrm{~Gy}$ fractions twice daily on days 1-5 of weeks $1,3,5,7 \pm 9$ for a total dose of 60-73.5 Gy. Concurrent systemic therapy consisted of 5 -FU $\left(600 \mathrm{mg} / \mathrm{m}^{2}\right), \mathrm{HU}(500 \mathrm{mg}$ BID) and cetuximab $\left(250 \mathrm{mg} / \mathrm{m}^{2}\right)$. p16 ${ }^{\mathrm{INK} 4 \mathrm{~A}}$ expression was assessed by immunohistochemistry. From January 2007 to January 2010, 65 patients (61 with stage IV disease; 31 with oropharyngeal primaries) were enrolled. At a median follow-up of 28 months, 2-year locoregional control, distant control, progression-free survival, event-free survival and overall survival were 79, 83, 72, 63 and $80 \%$, respectively. In 48 patients with available pre-treatment tissue, p16 overexpression was associated with significantly increased distant control $(\mathrm{p}=0.03)$, progression-free survival $(\mathrm{p}=0.02)$, event-free survival $(\mathrm{p}=0.007)$ and overall survival $(\mathrm{p}=0.03)$. The most common grade 3-4 toxicities were mucositis $(46 \%)$, leukopenia (18\%), anemia (18\%) and dermatitis
\end{abstract}

Correspondence to: Dr Charles C.L. Tong, Department of Radiation Oncology, and Department of Otolaryngology-Head and Neck Surgery, Mount Sinai School of Medicine, 1 Gustave Levy Place, Box 1189, New York, NY 10029, USA

E-mail: charles.tong@mssm.edu

"Contributed equally

Key words: cetuximab, chemoradiation, intensity modulated radiation therapy, head and neck cancer, epidermal growth factor receptor
(17\%). Concurrent 5-FU, HU, cetuximab and SIB-IMRT is a highly active regimen, particularly in patients with p16-positive disease.

\section{Introduction}

Concurrent chemoradiation is a standard treatment option for most patients with stage III-IVB head and neck cancer (1). Although concurrent high dose cisplatin $\left(100 \mathrm{mg} / \mathrm{m}^{2} \mathrm{q} 21\right.$ days $)$ is the best studied regimen, accumulating data suggests that either concurrent cetuximab ( $250 \mathrm{mg} / \mathrm{m}^{2} \mathrm{q} 7$ days) or 5-fluorouracil (5-FU) and hydroxyurea (HU) are also effective radiosensitizing regimens (2-5). Recent data suggests that adding induction chemotherapy to concurrent chemoradiation contributes to improved distant control and survival $(6,7)$. Although aggressive chemoradiation regimens contributed to promising rates of disease control, significant rates of acute and late toxicity have motivated clinical investigation of biologically targeted agents that hold promise for specific antitumor efficacy with less toxicity than chemotherapy. Epidermal growth factor receptor (EGFR) is overexpressed in $80-90 \%$ of patients with head and neck squamous cell carcinoma (HNSCC) and increased EGFR is associated with increased risk of disease progression and mortality following radiotherapy (8). Adding cetuximab to head and neck radiotherapy improves locoregional control and survival without increasing mucosal toxicity. Based on this compelling preclinical and clinical data, there is significant interest in incorporating cetuximab, a monoclonal antibody targeted against EGFR, into existing chemoradiation regimens (8).

To date, several groups have reported phase II trials demonstrating feasibility and encouraging preliminary efficacy data when EGFR inhibitors were added to cisplatin-based chemoradiotherapy regimens (9-12). A recent phase II trial demonstrated promising 4-year progression-free survival of $72 \%$ after treatment with induction carboplatin and paclitaxel followed by concurrent 5-FU, HU, gefitinib and hyperfractionated radiotherapy followed by maintenance gefitinib (11). Based on these encouraging data, we hypothesized that cetuximab, a targeted agent approved for use in HNSCC, 
would further improve outcomes without increasing toxicity. We have previously reported preliminary efficacy results (13).

Despite the high rate of EGFR overexpression, only 10\% of patients respond to EGFR inhibitors, and no prognostic factor can predict the response to EGFR-based therapy in HNSCC $(8,14,15)$. Approximately $25 \%$ of head and neck squamous cell carcinoma (HNSCC) cases worldwide are associated with high-risk human papillomaviruses (HPV) $16,18,31,33$ and 35 , with HPV-16 accounting for $\geq 90 \%$ of HPV-positive oropharyngeal cancer (16). Although data on the HPV infection status was not available in the phase III trial reported by Bonner et al, analysis of the Forrest plots suggests that the benefit of cetuximab occurs predominantly in younger patients with small oropharyngeal primary tumors with advanced nodal disease (3). This patient population has been strongly associated with high-risk HPV infection (17). $\mathrm{p} 16^{\mathrm{INK} 4 \mathrm{~A}}$ is a validated surrogate marker for overexpression of HPV-16 and has been extensively investigated as a prognostic factor for head and neck cancer patients treated with radiation or chemoradiation (18-20). Therefore, we evaluated a second hypothesis that p16-positive tumors are more likely to respond to cetuximab-based chemoradiation than p16-negative tumors.

\section{Patients and methods}

Ethics statement. Two phase II trials (MSSM 06-1155; NCT00462735) were approved by the Mount Sinai School of Medicine Institutional Review Board. All patients signed written informed consent.

Study design and eligibility. All patients had histologically confirmed head and neck squamous cell carcinoma or poorly differentiated carcinoma. Eligible patients had stage IVA-IVB disease according to the 6th edition of the AJCC staging guidelines, or high-risk stage III disease, defined as base of tongue or hypopharynx primary or major pathological risk factors (microscopic positive margins or extracapsular extension). Patients were required to have an ECOG performance status of $\leq 2$ and adequate bone marrow, kidney and liver function based on pre-treatment laboratory evaluation. All cases were reviewed at a multidisciplinary conference attended by representatives from head and neck surgery, radiation oncology, medical oncology, palliative care, social work and nutrition. Organ preserving surgery, defined as selective neck dissection or preservation of tongue, larynx, orbit, mandible or facial nerve and/or induction chemotherapy was allowed prior to registration on the protocol. Patients who received prior head and neck radiation were ineligible (Fig. 1).

Initial staging procedures included history and physical, nasolaryngoscopy and biopsy, dental evaluation, head and neck computed tomography $(\mathrm{CT})$ and chest $\mathrm{CT}$ with or without positron emission tomography (PET). Prophylactic feeding tubes were strongly recommended for patients with oral cavity involvement, massive tumors, advanced age or limited physiological reserve. Patients were followed prospectively by the multidisciplinary team usually with PET/CT-based follow-up (21).

Week on/week off (WO/WO) chemoradiotherapy. Patients received concurrent chemoradiotherapy consisting of continuous infusion $5-\mathrm{FU}$ at $600 \mathrm{mg} / \mathrm{m}^{2} /$ day $\times 120 \mathrm{~h}$ (days $1-5$ ) and

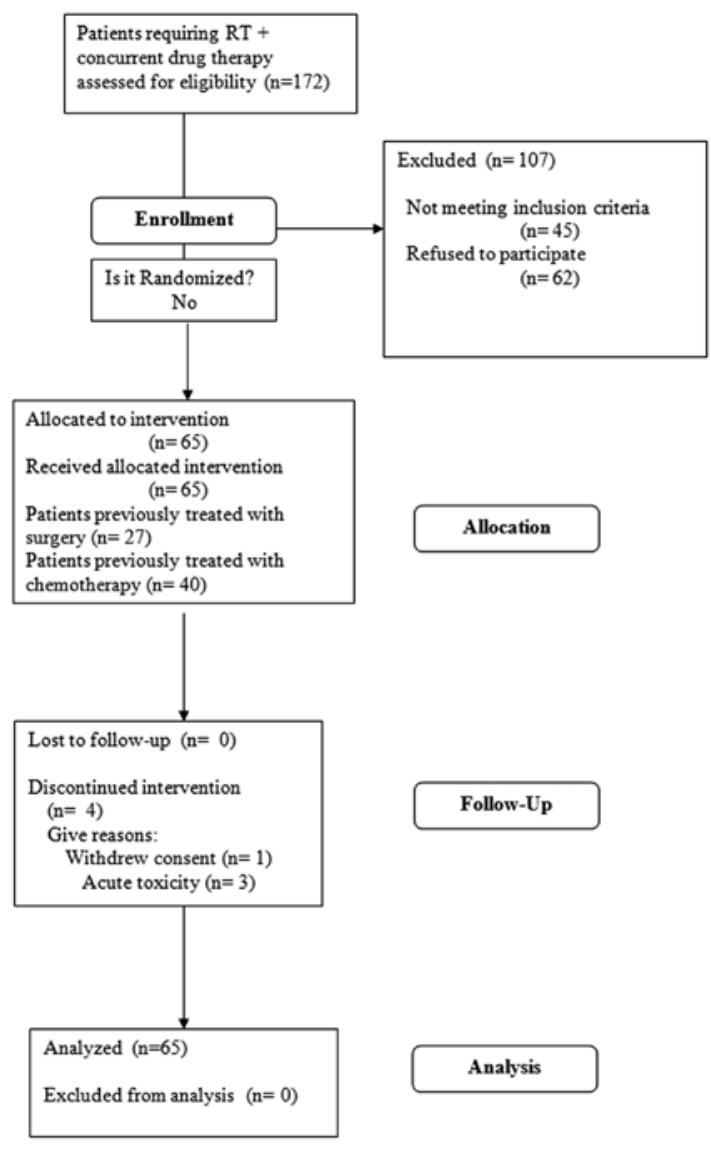

Figure 1. CONSORT flowchart.

HU $500 \mathrm{mg}$ orally q12 hours (days 1-5) with the morning dose administered $2 \mathrm{~h}$ before radiation and cetuximab $250 \mathrm{mg} / \mathrm{m}^{2}$ (day 1) on 14-day treatment cycles. Patients with gross disease received 5 cycles of chemoradiation, whereas post-surgical patients with microscopic disease received 4 cycles. Treatment was administered on an inpatient basis. For the first 33 patients, no anticancer therapy was administered on days 6-14. Based on the favorable toxicity profile seen in the initial cohort, the protocol was modified to add cetuximab $250 \mathrm{mg} / \mathrm{m}^{2}$ on day 9 for the remaining 32 patients (13). Concurrent chemoradiotherapy was withheld only for absolute neutrophil count (ANC) $<500 / \mu 1$, fever, infection or patient refusal but not for grade $\leq 3$ mucositis or dermatitis. The cetuximab dose was reduced by $20 \%$ for creatinine clearance $<50 \mathrm{ml} / \mathrm{min}$ or grade $\geq 3$ asthenia or nausea/vomiting attributed to cetuximab. Grade $\geq 3$ leukopenia or platelet resulted in a reduction in $\mathrm{HU}$ in subsequent cycles. Filgrastim $5 \mu \mathrm{g} / \mathrm{kg}$ was given by daily subcutaneous injection on days 6 through 12 following prior grade $\geq 3$ neutropenia.

Radiotherapy was administered at 1.5 Gy per fraction twice daily with fractions separated by at least $6 \mathrm{~h}$ on days $1-5$ on an alternating weekly schedule. Intensity-modulated radiation therapy (IMRT) was utilized for all patients. Patients underwent CT simulation, usually with fusion of PET or MRI imaging to assist with target delineation. When applicable, pre-induction chemotherapy tumor volumes were targeted.

Gross tumor volumes received 72 to $73.5 \mathrm{~Gy}$, microscopic positive margins received $66 \mathrm{~Gy}$, high-risk microscopic 
Table I. Patient, tumor and treatment characteristics.

\begin{tabular}{|c|c|}
\hline Characteristic & No. $(\%)$ \\
\hline \multicolumn{2}{|l|}{ Induction chemotherapy } \\
\hline Yes & $40(62)$ \\
\hline No & $25(38)$ \\
\hline \multicolumn{2}{|l|}{ Surgery prior to chemoRT } \\
\hline Yes & $27(42)$ \\
\hline No & $38(58)$ \\
\hline \multicolumn{2}{|l|}{ Cetuximab schedule } \\
\hline Biweekly & $33(51)$ \\
\hline Weekly & $32(49)$ \\
\hline Median age; range & $59(18-80)$ \\
\hline \multicolumn{2}{|l|}{ Gender } \\
\hline Male & $50(77)$ \\
\hline Female & $15(23)$ \\
\hline \multicolumn{2}{|l|}{ Performance status } \\
\hline 0 & $20(31)$ \\
\hline 1 & $33(51)$ \\
\hline 2 & $12(18)$ \\
\hline \multicolumn{2}{|l|}{ Race } \\
\hline White & $49(75)$ \\
\hline Black & $7(11)$ \\
\hline Hispanic & $7(11)$ \\
\hline Asian & $2(3)$ \\
\hline \multicolumn{2}{|l|}{ Smoking history } \\
\hline None & $14(22)$ \\
\hline Cigar, pipe, marijuana or betel nut only & $5(8)$ \\
\hline$\leq 10$ pack years & $12(18)$ \\
\hline 10.1-40 pack years & $19(29)$ \\
\hline$\geq 40$ pack years & $15(23)$ \\
\hline \multicolumn{2}{|l|}{ Primary site } \\
\hline Sinonasal & $3(5)$ \\
\hline Nasopharynx & $5(8)$ \\
\hline Oropharynx & $31(48)$ \\
\hline Oral cavity & $8(12)$ \\
\hline Salivary gland & $2(3)$ \\
\hline Larynx & $5(8)$ \\
\hline Hypopharynx & $7(11)$ \\
\hline Unknown primary & $4(6)$ \\
\hline \multicolumn{2}{|l|}{ AJCC stage } \\
\hline III & $4(6)$ \\
\hline IVA & $50(77)$ \\
\hline IVB & $11(17)$ \\
\hline \multicolumn{2}{|l|}{ T stage } \\
\hline $\mathrm{T} 0-2$ & $29(45)$ \\
\hline Т 3-4 & $36(55)$ \\
\hline \multicolumn{2}{|l|}{$\mathrm{N}$ stage } \\
\hline No & $9(14)$ \\
\hline $\mathrm{N} 1$ & $9(14)$ \\
\hline $\mathrm{N} 2$ & $42(65)$ \\
\hline $\mathrm{N} 3$ & $5 \quad(8)$ \\
\hline \multicolumn{2}{|l|}{ p16 status } \\
\hline Negative & $26(40)$ \\
\hline Positive $w / \leq 10$ pack years & $12(28)$ \\
\hline Positive $w />10$ pack years & $10(15)$ \\
\hline Unknown & $17(26)$ \\
\hline
\end{tabular}

disease (resected tumor bed or first echelon of uninvolved nodal stations) received 54 to $63 \mathrm{~Gy}$ and low-risk microscopic disease (low-risk nodal stations) received 43.2 to $48 \mathrm{~Gy}$. The microscopic volumes were treated with a simultaneous integrated boost plan. Radiation dose constraints were described previously (13). A separate cone down was performed for patients with gross disease. Patients received the lower dose levels if they achieved a partial or complete response to induction chemotherapy. The median dose was $72 \mathrm{~Gy}$ (range 13.5 to $73.5 \mathrm{~Gy}$ ). The median duration of the treatment was 60 days (range 5 to 87 days).

Induction chemotherapy. Induction chemotherapy, given off protocol, was administered to selected patients at the discretion of the treating medical oncologist (Table I). The most common indication for induction chemotherapy was high-risk of distant failure due to advanced N2b to N3 nodal disease. Measurable disease was not considered a requirement for induction chemotherapy. Docetaxel $\left(75 \mathrm{mg} / \mathrm{m}^{2}\right)$, cisplatin $\left(75 \mathrm{mg} / \mathrm{m}^{2}\right)$ and $5-\mathrm{FU}$ ( $750 \mathrm{mg} / \mathrm{m}^{2} \times 5$ days) $\mathrm{q} 3$ weeks for 2 cycles was given to 40 patients $(62 \%)$.

Surgery. Organ-conserving surgery was performed in 27 patients (42\%) prior to chemoradiation (Table I). This included 16 patients treated with resection of the primary site and selective neck dissection, 3 patients with resection of the primary site alone and 8 patients with neck dissection alone. Adverse pathology included 14 patients with microscopic positive margins, 14 patients with perineural invasion, 9 patients with lymphovascular invasion, 15 patients with multiple pathologically positive nodes and 18 patients with extracapsular extension. Selective neck dissection after complete response of N2-3 nodal disease following chemoradiation was performed in 6 patients.

p16 ${ }^{I N K 4 A}$ immunohistochemistry. Tumor blocks were available in 48 pre-treatment patients $(74 \%)$. Briefly, tissues were fixed in $10 \%$ formalin, embedded in paraffin and $5 \mu \mathrm{m}$ sections were adhered to charged glass slides. Slides were deparaffinized in a $60^{\circ} \mathrm{C}$ oven for $30 \mathrm{~min}$ prior to xylene bath, and rehydrated with ethyl alcohol. Prior to immunostaining, epitope retrieval was achieved by incubating slides in retrieval solution placed in a $97^{\circ} \mathrm{C}$ water bath for $10 \mathrm{~min}$ and allowed to cool to room temperature for $20 \mathrm{~min}$. Endogenous peroxidase activity was blocked using peroxidase-blocking reagent followed by application of the primary mouse anti-human p16 $6^{\mathrm{INK} 4 \mathrm{~A}}$ antibody (mtm Laboratories, Westborough, MA) for $30 \mathrm{~min}$ at room temperature. The visualization reagent containing goat anti-mouse secondary antibody was applied for $30 \mathrm{~min}$ at room temperature followed by DAB chromogen for $1 \mathrm{~min}$. Counterstaining was achieved using Mayer's hematoxylin. After dehydration and mounting, slides were scored for p16 staining as negative (Fig. 3A), $1^{+}, 2^{+}$ and $3^{+}$by a single pathologist (M.R.) with subspecialty training in head and neck cancer pathology who was blinded to patient outcome. Scores of $2^{+}$to $3^{+}$were considered positive (Fig. 3B). Since p16 staining is routinely used in our clinical practice, previously scored patients with p16-positive and negative tumors were used as controls.

Treatment evaluation and statistical considerations. The primary endpoint was progression-free survival, measured 
A


B

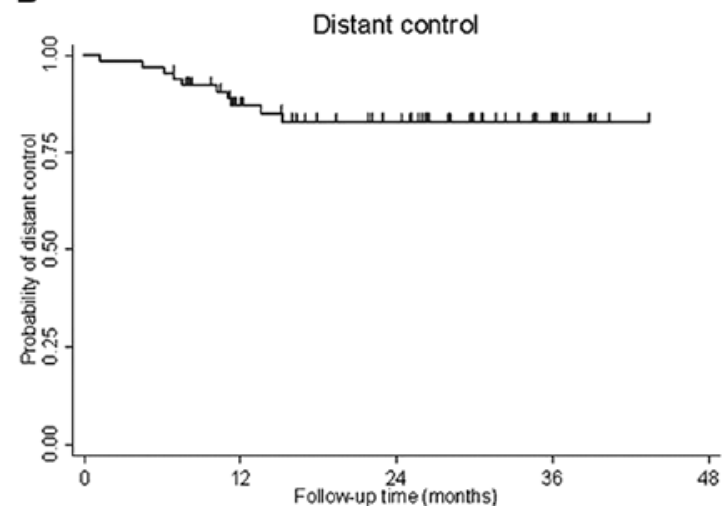

D

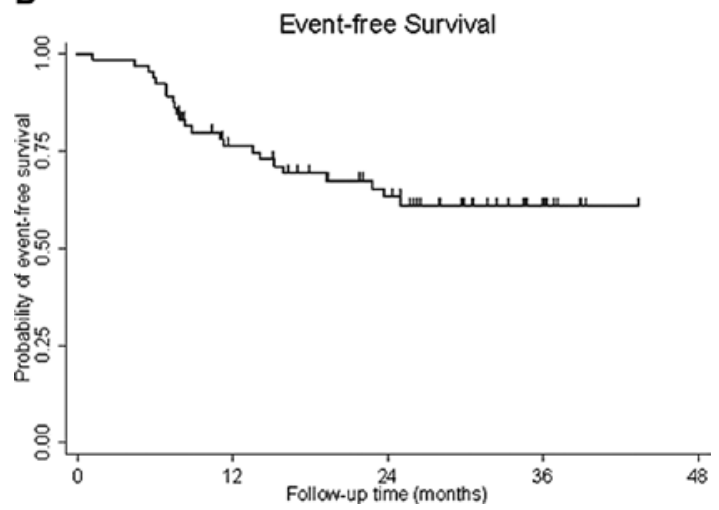

E

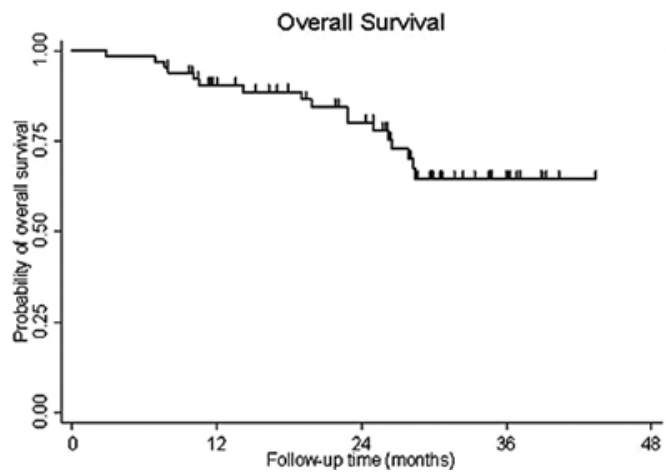

Figure 2. Kaplan-Meier survival curves: (A) locoregional control, (B) distant control, (C) progression-free survival, (D) event-free survival, (E) overall survival.

as time from the initiation of non-surgical treatment until last follow-up or disease progression using intent to treat principles. Failures were scored as local, regional or distant. Eventfree survival is a composite endpoint that includes disease progression, second primary tumor or death. Survival curves were calculated using Kaplan-Meier curves using Stata 9.1. Differences in survival curves were calculated by using the log-rank test. Univariate and multivariable analyses of patient and disease factors include age, race, smoking history, gender, performance status, stage, tumor site, p16, surgery, induction chemotherapy and frequency of cetuximab.

\section{Results}

Patient characteristics. From January 2007 to January 2010, 65 patients were enrolled (Table I). Median follow-up for surviving patients was 28 months (range $8-43$ months). The patient characteristics are summarized in Table I. The median age was 59 years (range 18-80). Sixty-one patients (94\%) had stage IV disease and 4 patients (6\%) had stage III disease, including 7 patients with radiographic evidence of progression following either surgery or induction chemotherapy prior to starting chemoradiation. Thirty-six patients $(55 \%)$ had T3-4 primary tumors and 18 patients $(27 \%)$ had N2-3 nodal disease (Table I).

Survival and patterns of failure. The 2-year locoregional control, distant control, progression-free survival, event-free survival and overall survival were 79, 83, 72, 63 and $80 \%$, respectively (Fig. 2). The 2-year local control rate was $85 \%$ and the 2-year regional control rate was $82 \%$. At the time this manuscript was prepared, 17 patients $(26 \%)$ had experienced 

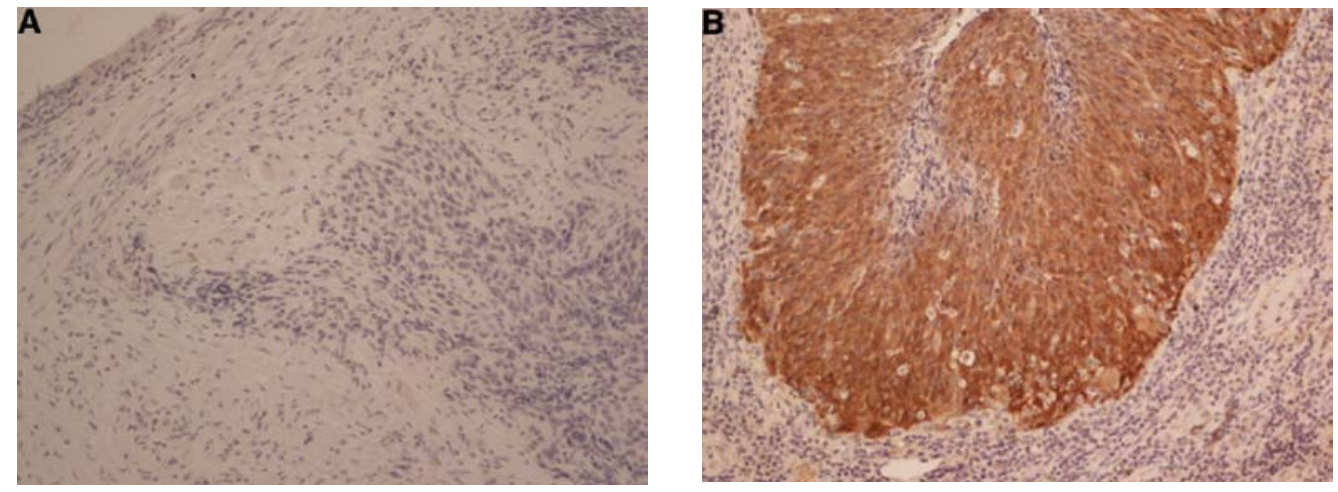

Figure 3. p16 immunostaining of pre-treatment tumor specimens at 20x magnification. (A) Note irregular infiltrates of tumor cells with absence of p16 staining (B) Note intense tumor-specific staining with nuclear enhancement and absence of staining in adjacent stroma.

Table II. Percentage of patients $(\mathrm{n}=65)$ with acute toxicity grade during concurrent chemoradiotherapy.

\begin{tabular}{lrrcc}
\hline Toxicity & Grade 1 & Grade 2 & Grade 3 & Grade 4 \\
\hline Mucositis & 11 & 40 & 46 & 2 \\
Dermatitis & 30 & 52 & 17 & 0 \\
Pain & 6 & 43 & 49 & 0 \\
ANC & 29 & 23 & 9 & 0 \\
WBC & 28 & 46 & 18 & 0 \\
Hgb & 35 & 43 & 18 & 0 \\
Plt & 31 & 0 & 2 & 0 \\
Nausea/vomiting & 8 & 3 & 0 & 0 \\
Infection & 2 & 6 & 2 & 3 \\
Xerostomia & 44 & 48 & 0 & 0 \\
Sialadenitis & 0 & 5 & 0 & 0 \\
Dry eye & 0 & 3 & 0 & 0 \\
\hline
\end{tabular}

ANC, absolute neutrophil count; WBC, white blood cells; Hgb, hemoglobin; Plt, platelets.

disease recurrence. Patterns of failure were locoregional only in 7, distant only in 5 and both locoregional and distant in 5 . Additionally, four patients developed second primary tumors; 12 patients had died of recurrent disease, 2 had died of second primary tumors and 2 patients had died of comorbid illnesses.

Effect of p16 status on disease control. Increased p16 expression was strongly associated with improved 2-year overall survival (94.4 vs. $69.5 \%$; $\mathrm{p}=0.03$ ), progression-free survival ( 86.4 vs. $54.3 \%$; $\mathrm{p}=0.02$ ), event-free survival ( 86.3 vs. $41.5 \%$; $\mathrm{p}=0.007)$, locoregional control (90.9 vs. $64.2 \% ; \mathrm{p}=0.05)$ and distant control ( 95.5 vs. $71.5 \%$; $\mathrm{p}=0.03$ ) on univariate analysis (Fig. 4). Patients with p16-positive tumors with a minimal smoking history ( $\leq 10$ pack years) had a significantly improved 2-year event-free survival ( 87.5 vs. $70.0 \%$; $\mathrm{p}=0.02$ ) compared to p16-positive smokers ( $>10$ pack years). Multivariable analysis demonstrated that p16 status, stratified by smoking status, was the only significant predictor of event-free survival [hazard ratio (HR), 0.43; $\mathrm{p}=0.04$ ] while there was a marginal effect for AJCC stage (HR, 4.1; $\mathrm{p}=0.09)$ (Table III).
Table III. Multivariable analyses.

\begin{tabular}{|c|c|c|c|}
\hline Factor & HR & $95 \% \mathrm{CI}$ & $\mathrm{p}$ \\
\hline Age (continuous) & 1.003 & $0.95-1.05$ & 0.89 \\
\hline Gender (female vs. male) & 0.65 & $0.20-2.1$ & 0.48 \\
\hline $\begin{array}{l}\text { Performance status } \\
(0 \text { vs. } 1 \text { vs. } 2)\end{array}$ & 1.70 & $0.68-4.27$ & 0.26 \\
\hline Race (black vs. white) & 2.14 & $0.72-6.41$ & 0.17 \\
\hline $\begin{array}{l}\text { p16 status (p16-, p16 } 16^{+} \text {smoker, } \\
\text { p16 }\end{array}$ & 0.43 & $0.19-0.95$ & 0.04 \\
\hline $\begin{array}{l}\text { Primary site (oropharynx } \\
\text { vs. non-oropharynx) }\end{array}$ & 0.78 & $0.23-2.61$ & 0.69 \\
\hline Stage (III, IVA, IVB) & 4.12 & $0.82-20.80$ & 0.09 \\
\hline Induction (yes vs. no) & 0.33 & $0.08-1.40$ & 0.13 \\
\hline Surgery (yes vs. no) & 0.73 & $0.24-2.21$ & 0.58 \\
\hline $\begin{array}{l}\text { Cetuximab schedule } \\
\text { (biweekly vs. weekly) }\end{array}$ & 0.73 & $0.22-2.47$ & 0.62 \\
\hline
\end{tabular}

$\mathrm{HR}$, hazard ratio; CI, confidence interval.

Effect of site-specific tumors and p16 status on disease control. Of the 48 patients with pre-treatment tissues available for 16 staining, 22 (46\%) were tumors from the oropharynx and 26 (54\%) were tumors from non-oropharyngeal sites. Increased p16 expression $(n=15)$ among the oropharyngeal group was strongly associated with overall survival ( $93 \mathrm{vs.} 43 \% ; \mathrm{p}=0.015$ ), locoregional control (100 vs. $43 \%$; $\mathrm{p}=0.001$ ), distant control ( 93 vs. $43 \%$; $\mathrm{p}=0.028$ ), progression-free survival ( 93 vs. $43 \%$; $\mathrm{p}=0.005$ ), and event-free survival (93 vs. $14 \%$; $\mathrm{p}=0.001$ ). Patients with p16-positive non-oropharyngeal tumors $(n=7)$ did not demonstrate statistically significant benefit in similar endpoints.

Adverse events. A percutaneous gastrostomy (PEG) tube was placed in $18 \%$ of patients before initiation of radiation while $52 \%$ of the patients ultimately required PEG, total parenteral nutrition or a jejunostomy tube during treatment. The median weight loss was $9.5 \%$ (range $0-17.4 \%$ ). The most common grade $\geq 3$ acute toxicities were mucositis (48\%), anemia (18\%), leukopenia (18\%), dermatitis (17\%) and neutropenia (9\%) (Table II). Other frequent acute low-grade treatment-related toxicities included fatigue, 
A

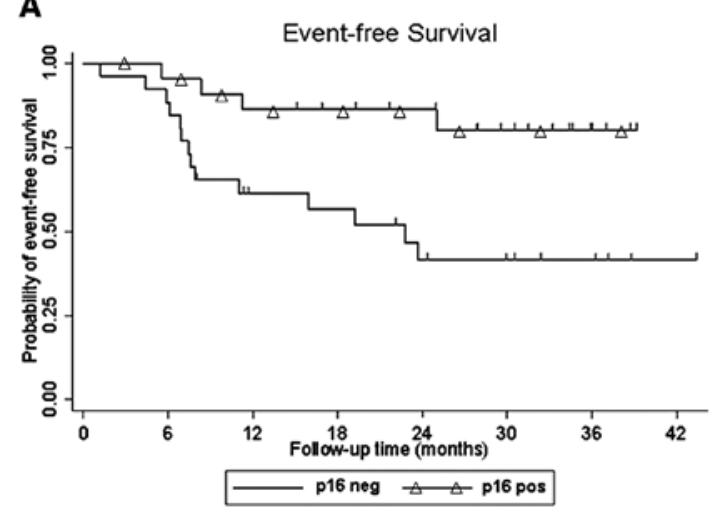

B

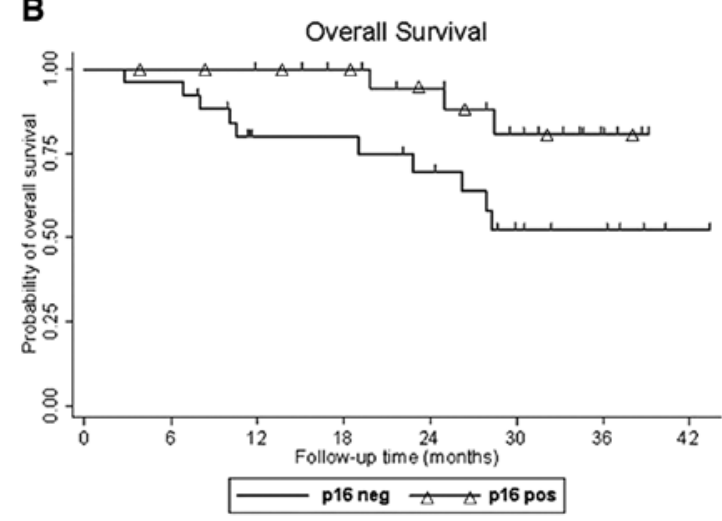

Figure 4. Kaplan-Meier survival curves stratified by p16 status: (A) event free survival, (B) overall survival.

xerostomia, taste changes and thrush. Although there were no grade 5 events or neutropenic infections, two patients developed grade 4 non-neutropenic aspiration pneumonia requiring intensive care unit (ICU) care. Both patients were ultimately moved from the ICU but discontinued radiation at doses of $13.5 \mathrm{~Gy}$ and $33 \mathrm{~Gy}$, respectively. Therefore, $97 \%$ of patients were able to complete $\geq 95 \%$ of the planned radiation dose. Other protocol deviations included 4 patients that had an unplanned treatment break of $\geq 7$ days due to toxicity or non-compliance and 1 patient who withdrew consent during chemoradiation and requested an alternative chemoradiation regimen.

Excluding patients with nutritional problems related to recurrent disease, only 2 patients required prolonged use of a feeding tube $\geq 1$ year. Other grade 2-3 late toxicities included xerostomia $(n=12)$, bone necrosis $(n=3)$, soft tissue necrosis or ulceration $(n=3)$, esophageal stricture $(n=3)$, skin ulceration $(n=1)$, trismus $(n=1)$ and dysphagia to solid foods $(n=5)$. Two of these patients required reconstructive surgery while the other adverse events were treated conservatively.

\section{Discussion}

This report of a single institution phase II trial demonstrates that concurrent 5-FU, HU, cetuximab and hyperfractionated IMRT is a feasible and active regimen in stage III-IVB HNSCC. Despite this complex regimen, often preceded by induction chemotherapy or surgery, approximately $30 \%$ of treated patients progressed. The 2-year locoregional control was $79 \%$ while the 2-year distant control was $83 \%$. Prior phase II trials performed at the University of Chicago consisting of induction chemotherapy followed by 5-FU, hydroxyurea, paclitaxel and hyperfractionated RT demonstrated rates of locoregional control and distant control in the $90 \%$ range (22-24). Although a randomized trial is necessary to test this hypothesis, these data suggest that cetuximab-based chemoradiation may be slightly less effective than an intensive paclitaxel or cisplatinbased regimen. Overall, progression-free survival following treatment with concurrent 5-FU, hydroxyurea, cetuximab and hyperfractionated IMRT compares favorably with other published chemoradiation regimens $(7,8,25,26)$.

Although 5-FU, HU, paclitaxel and hyperfractionated radiation demonstrates robust antitumor activity, a significant subset of patients treated with this intensive regimen succumbed to death attributed to toxicity or comorbid illness (27). Numerous strategies to reduce acute and chronic toxicity have been examined, including using IMRT in lieu of conventional radiation, reducing radiation doses in responders to induction chemotherapy and using EGFR-inhibitors instead of paclitaxel $(11,28,29)$. Although the marginal benefit of each individual strategy was difficult to detect, implementing all three approaches together in this trial appeared to reduce the incidence of treatment-related toxicity and death compared to prior studies (13). A key advantage of 5-FU, hydroxyurea, cetuximab and hyperfractionated IMRT are low rates of myelosuppression and neutropenic infection. A clear disadvantage is the significant logistical challenges associated with inpatient administration of chemoradiation that includes twice-daily IMRT. As a result, identifying a cohort that benefits from this non-standard but effective strategy for locally advanced HNSCC would guide clinical decisions and future research directions.

Human papillomavirus-16 is an important etiologic and prognostic factor for HNSCC (17). HPV-related tumors have significantly improved locoregional control and survival when treated with radiation alone, concurrent chemoradiation, concurrent chemotherapy and accelerated radiation, induction chemotherapy followed by chemoradiation or surgery $(19,25,30,31)$. To date, it is not known whether HPV-related tumors are more likely to respond to EGFR inhibitors (15). In this study of patients treated with cetuximab-based chemoradiation, patients with p16-positive tumors had significantly improved overall survival, event-free survival, progression-free survival, locoregional control and distant control compared to p16-negative tumors. Although not definitive, these data suggest p16-positive oropharyngeal tumors respond much more favorably to cetuximab-based chemoradiation. More effective treatment for the HPV-negative cohort represents a high priority unmet need for future research. To date, this cohort appears to be refractory to various treatment intensification strategies including sequential chemotherapy followed by concurrent chemotherapy and accelerated fractionation radiation enhanced by concurrent chemotherapy $(25,30)$.

Preclinical data suggests that cetuximab-mediated enhancement of radiotherapy may be dose-dependent (32). Interestingly, there was no significant difference in the outcome detected in this study whether or not patients received additional cetuximab during non-radiation weeks. In the study 
of Bonner et al, concurrent cetuximab and radiation improved locoregional control compared to radiation alone without improving distant control (8). Taken together, the data suggest that short course cetuximab given during radiotherapy is ineffective at targeting micrometastases. The role of maintenance treatment with EGFR-inhibitors to improve progression-free survival is under active investigation (11).

In conclusion, concurrent 5-FU, hydroxyurea, cetuximab and hyperfractionated IMRT is an effective approach for the treatment of locoregionally advanced HNSCC. Despite aggressive treatment, patients with p16-negative tumors continue to have a poor prognosis.

\section{Acknowledgements}

This study was funded in part by the Mount Sinai School of Medicine. The funders had no role in study design, data collection and analysis, decision to publish or preparation of the manuscript. J.K. received research funding from OSI Pharmaceuticals. S.P. and J.K. were members of the Speakers' Bureau for Sanofi-Aventis. The authors have declared that no competing interests exist.

\section{References}

1. Haddad RI and Shin DM: Recent advances in head and neck cancer. N Engl J Med 359: 1143-1154, 2008.

2. Adelstein DJ, Li Y, Adams GL, et al: An intergroup phase III comparison of standard radiation therapy and two schedules of concurrent chemoradiotherapy in patients with unresectable squamous cell head and neck cancer. J Clin Oncol 21: 92-98, 2003.

3. Bonner JA, Harari PM, Giralt J, et al: Radiotherapy plus cetuximab for locoregionally advanced head and neck cancer: 5 -year survival data from a phase 3 randomised trial, and relation between cetuximab-induced rash and survival. Lancet Oncol 11: 21-28, 2010.

4. Forastiere AA, Goepfert H, Maor M, et al: Concurrent chemotherapy and radiotherapy for organ preservation in advanced laryngeal cancer. N Engl J Med 349: 2091-2098, 2003.

5. Garden AS, Harris J, Vokes EE, et al: Preliminary results of Radiation Therapy Oncology Group 97-03: a randomized phase ii trial of concurrent radiation and chemotherapy for advanced squamous cell carcinomas of the head and neck. J Clin Oncol 22: 2856-2864, 2004.

6. Brockstein B, Haraf DJ, Rademaker AW, et al: Patterns of failure, prognostic factors and survival in locoregionally advanced head and neck cancer treated with concomitant chemoradiotherapy: a 9-year, 337-patient, multi-institutional experience. Ann Oncol 15: 1179-1186, 2004.

7. Posner MR, Hershock DM, Blajman CR, et al: Cisplatin and fluorouracil alone or with docetaxel in head and neck cancer. $\mathrm{N}$ Engl J Med 357: 1705-1715, 2007.

8. Bonner JA, Harari PM, Giralt J, et al: Radiotherapy plus cetuximab for squamous-cell carcinoma of the head and neck. $\mathrm{N}$ Engl J Med 354: 567-578, 2006.

9. Merlano M, Russi E, Benasso M, et al: Cisplatin-based chemoradiation plus cetuximab in locally advanced head and neck cancer: a phase II clinical study. Ann Oncol 22: 712-717, 2010.

10. Pfister DG, Su YB, Kraus DH, et al: Concurrent cetuximab, cisplatin, and concomitant boost radiotherapy for locoregionally advanced, squamous cell head and neck cancer: a pilot phase II study of a new combined-modality paradigm. J Clin Oncol 24: 1072-1078, 2006.

11. Cohen EE, Haraf DJ, Kunnavakkam R, et al: Epidermal growth factor receptor inhibitor gefitinib added to chemoradiotherapy in locally advanced head and neck cancer. J Clin Oncol 28: 3336-3343, 2010.

12. Harrington KJ, El-Hariry IA, Holford CS, et al: Phase I study of lapatinib in combination with chemoradiation in patients with locally advanced squamous cell carcinoma of the head and neck J Clin Oncol 27: 1100-1107, 2009.
13. Kao J, Genden EM, Gupta V, et al: Phase 2 trial of concurrent 5-fluorouracil, hydroxyurea, cetuximab, and hyperfractionated intensity-modulated radiation therapy for locally advanced head and neck cancer. Cancer 117: 318-326, 2011.

14. Cohen EE, Rosen F, Stadler WM, et al: Phase II trial of ZD1839 in recurrent or metastatic squamous cell carcinoma of the head and neck. J Clin Oncol 21: 1980-1987, 2003.

15. Eriksen JG, Lassen P and Overgaard J: Do all patients with head and neck cancer benefit from radiotherapy and concurrent cetuximab? Lancet Oncol 11: 312-313, 2010.

16. Kreimer AR, Clifford GM, Boyle P, et al: Human papillomavirus types in head and neck squamous cell carcinomas worldwide: a systematic review. Cancer Epidemiol Biomarkers Prev 14: 467-475, 2005.

17. Gillison ML: Human papillomavirus and prognosis of oropharyngeal squamous cell carcinoma: implications for clinical research in head and neck cancers. J Clin Oncol 24: 5623-5625, 2006.

18. Shi W, Kato H, Perez-Ordonez B, et al: Comparative prognostic value of HPV16 E6 mRNA compared with in situ hybridization for human oropharyngeal squamous carcinoma. J Clin Oncol 27: 6213-6221, 2009.

19. Lassen P, Eriksen JG, Hamilton-Dutoit S, et al: HPV-associated p16-expression and response to hypoxic modification of radiotherapy in head and neck cancer. Radiother Oncol 94: 30-35, 2010.

20. Weinberger PM, Yu Z, Haffty BG, et al: Molecular classification identifies a subset of human papillomavirus - associated oropharyngeal cancers with favorable prognosis. J Clin Oncol 24: 736-747, 2006.

21. Kao J, Vu HL, Genden EM, et al: The diagnostic and prognostic utility of positron emission tomography/computed tomographybased follow-up after radiotherapy for head and neck cancer. Cancer 115: 4586-4594, 2009.

22. Michiels S, Le Maitre A, Buyse M, et al: Surrogate endpoints for overall survival in locally advanced head and neck cancer: metaanalyses of individual patient data. Lancet Oncol 10: 341-350, 2009.

23. Haraf DJ, Rosen FR, Stenson K, et al: Induction chemotherapy followed by concomitant TFHX chemoradiotherapy with reduced dose radiation in advanced head and neck cancer. Clin Cancer Res 9: 5936-5943, 2003.

24. Vokes EE, Stenson K, Rosen FR, et al: Weekly carboplatin and paclitaxel followed by concomitant paclitaxel, fluorouracil, and hydroxyurea chemoradiotherapy: curative and organ-preserving therapy for advanced head and neck cancer. J Clin Oncol 21: 320-326, 2003.

25. Ang KK, Harris J, Wheeler R, et al: Human papillomavirus and survival of patients with oropharyngeal cancer. N Engl J Med 363: 24-35, 2010

26. Brizel DM, Albers ME, Fisher SR, et al: Hyperfractionated irradiation with or without concurrent chemotherapy for locally advanced head and neck cancer. N Engl J Med 338: 1798-1804, 1998.

27. Mell LK, Dignam JJ, Salama JK, et al: Predictors of competing mortality in advanced head and neck cancer. J Clin Oncol 28: 15-20, 2010.

28. Milano MT, Vokes EE, Kao J, et al: Intensity-modulated radiation therapy in advanced head and neck patients treated with intensive chemoradiotherapy: preliminary experience and future directions. Int J Oncol 28: 1141-1151, 2006.

29. Salama JK, Stenson KM, Kistner EO, et al: Induction chemotherapy and concurrent chemoradiotherapy for locoregionally advanced head and neck cancer: a multi-institutional phase II trial investigating three radiotherapy dose levels. Ann Oncol 19: 1787-1794, 2008.

30. Fakhry C, Westra WH, Li S, et al: Improved survival of patients with human papillomavirus-positive head and neck squamous cell carcinoma in a prospective clinical trial. J Natl Cancer Inst 100: 261-269, 2008.

31. Licitra L, Perrone F, Bossi P, et al: High-risk human papillomavirus affects prognosis in patients with surgically treated oropharyngeal squamous cell carcinoma. J Clin Oncol 24: 5630-5636, 2006.

32. Milas L, Fang FM, Mason KA, et al: Importance of maintenance therapy in C225-induced enhancement of tumor control by fractionated radiation. Int J Radiat Oncol Biol Phys 67: 568-572, 2007. 